\title{
Sabine Höflich
}

Pädagogische Hochschule Niederösterreich, Campus Baden

im Gespräch mit

\section{Astrid Standl-Wimmer}

Volksschule Breitenfurt

\section{Resilient in herausfordernden Zeiten?}

\section{Ein Gespräch über die stärkende Bedeutung von Kontakt in Zeiten der Kontaktreduktion}

DOI: https://doi.org/10.53349/sv.2021.i3.a137

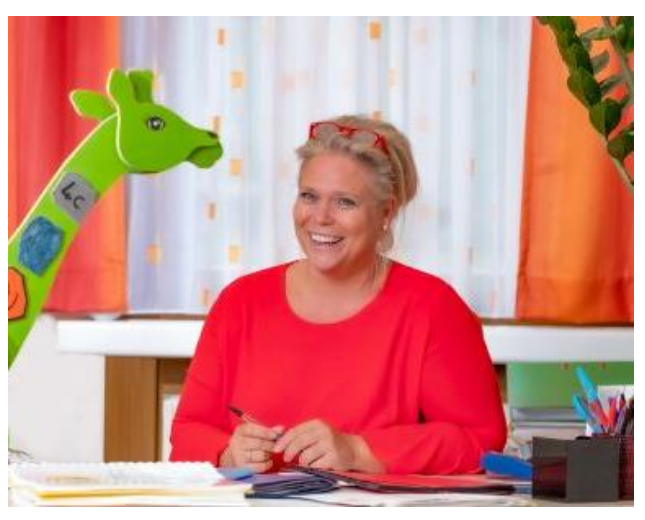

sp
Astrid Standl-Wimmer, MA BEd.

Schulleiterin der Volksschule Breitenfurt bei Wien Lehramt für Volksschulen, Masterstudium an der Donau-Uni Krems mit dem Schwerpunkt Gewaltprävention und Gewaltfreie Kommunikation, Qualifikationen im Bereich Schulmanagement.

„Mein Name ist Astrid Standl-Wimmer. Ich bin 47 Jahre alt, verheiratet und Mutter eines erwachsenen Sohnes und einer 7-jährigen Tochter. Seit 24 Jahren bin ich mit Herz und Seele im Schuldienst tätig, zuerst als klassenführende Pädagogin und seit 2019 als Schulleiterin der Volksschule Breitenfurt. Schulleben mitgestalten und Schulqualität mitentwickeln zu dürfen, sind für mich spannende, großartige Aufgaben und in dieser Zeit eine besondere Herausforderung."

HOFFNUNGsschule als stärkendes Miteinander

Im Gespräch über Resilienz, der psychischen Widerstandsfähigkeit, starke Kinder und erfolgreiche Teams berichtet Schulleiterin Astrid Wimmer-Standl von der Bedeutung des Miteinanders, das Hoffnung gibt und stark machen kann. 
„Es ist viel passiert, obwohl nicht viel passieren durfte“, meint Astrid Standl-Wimmer im Gespräch über Optimismus, Vorbilder und Stärkung der psychischen Widerstandsfähigkeit der Resilienz kurz vor dem vierten Lockdown im November 2021. Auch in Zeiten von Lockdowns, Schichtbetrieb und Kontaktbeschränkung wurden Wege gefunden, um in Kontakt zu bleiben und die Erfahrung, diese herausfordernde, von Veränderung und Unbekanntem gekennzeichneten Zeiten, bewältigt zu haben, soll Hoffnung und Zuversicht bieten, auch diesmal zu guten Lösungen zu kommen.

Sechs Monate vor dem Beginn der Pandemie, als sie 2019 die Schulleitung der Volksschule übernommen hatte, machte sie gemeinsam mit ihrem Team die „Gemeinsam stark werden das Lebenskompetenzprogramm für die Volksschule"-Fortbildung. Dies ist ein suchtpräventives Fortbildungsprogramm für Grundschullehrer*innen mit dem Ziel der Stärkung der Lebenskompetenzen, welche als Voraussetzung für ein gesundes, selbstbestimmtes und erfülltes Leben beschrieben wird (vgl. Fachstelle NÖ, 2021).

\section{Gibt es auch andere Maßnahmen, vielleicht auch aufgrund von Corona, die Sie gerne um- setzen wollen? Oder sagen Sie, dieses Projekt führt durch sämtliche Krisen?}

Astrid Standl-Wimmer: Dieses Projekt alleine führt uns nicht durch alle Krisen, jedoch hat es einen gesunden Teambuilding-Prozess eingeleitet und fördert unsere Freude, unseren Mut und Tatendrang rund um unser pädagogisches Tun und Handeln. Die Umsetzung des Projektes hatte nicht nur resilienzfördernde Maßnahmen und Unterstützung für unsere Kinder im Fokus, sondern gab uns Pädagog*innen wertvolle Ideen, die eigene Resilienz in dieser herausfordernden Zeit der Pandemie zu stärken. Das soziale Lernen im Rahmen des Schulalltages ist seit Beginn der Pandemie bedeutsamer denn je und braucht genügend Zeit und Raum. Die auftretenden Sorgen verbalisieren zu lernen, Gefühle zeigen und einordnen zu können, sind sowohl für unsere Schüler*innen als auch für Eltern und uns Pädagog*innen von großer Bedeutung. Maßnahmen, wie die Komplimente-Runde, Sonnendusche, der Sorgenbriefkasten, unser Kinderparlament oder auch die Schulstreitschlichter*innen sind bereits ein fixer Bestandteil des Klassen- und Schulalltages.

In den ersten Monaten des Schuljahres 2021/22 starteten wir eine Kooperation mit dem Roten Kreuz. Acht Wochen lang waren Schul-Therapiehunde zu Besuch mit dem Ziel, individuelle, soziale Kompetenzen und die kindliche Gefühlswelt zu stärken. Der Erfolg war schon nach den ersten Stunden zu sehen. 


\section{Was braucht Schule, damit sie zum resilienzförderlichen Ort wird?}

Eine resilienzfördernde Schule fällt durch ein positives Schulklima auf, ist gekennzeichnet von vertrauensvollen Begegnungen, gesundem Wachsendürfen und respektvollem Umgang mit Stärken und Schwächen. Eine resiliente Schule soll ein Ort sein, an dem man akzeptiert wird, sich selbstwirksam erlebt und klare Regeln und Strukturen Sicherheit geben. Dafür braucht es in der Schule eine gute Beziehungsgestaltung, die Vertrauen schafft und fördert. Die Pandemie hat uns gezeigt, wie wichtig diese besonderen Beziehungen zu Schulfreund*innen und Lehrer*innen für heranwachsende Kinder sind und wie sehr diese im „Distanzlernen“ fehlen können.

Kurz zusammenfasst: Eine resiliente Schule ist für mich ein Ort, wo ich gerne lerne, wo ich gerne bin, wo ich gesehen und wahrgenommen werde!

Haben Sie in dieser Schilderung die Kinder im Fokus gehabt oder haben Sie auch an Ihr Lehrer*innenteam gedacht?

Sowohl als auch, mein erster Gedanke galt den Kindern. Natürlich ist es mir außerdem wichtig, dass unsere Schule ein resilienzförderlicher Ort für mein Team ist. Wir müssen uns nur im Klaren sein, dass die Gestaltung in der Verantwortung aller Erwachsenen liegt.

\section{Was machen Sie selbst, um stark und resilient zu sein und auch zu bleiben?}

Momentan sind wir in einer turbulenten Zeit, in der uns eine gewisse Gefühlsstabilität enorm hilfreich ist. Ich bin grundsätzlich ein sehr optimistischer und positiv denkender Mensch und schaue mit Zuversicht in die Zukunft. Auch gewisse Denkpfade zu verlassen und den Blick auf Situationen aus mehreren Perspektiven zu richten, hilft enorm - ist nicht immer einfach, gelingt meistens. Eine Portion Humor und Gelassenheit sind in manchen Situationen ebenso notwendig. Um weiterhin stark und resilient zu bleiben, freue ich mich über Zeit und Gespräche mit meiner Familie und Freund*innen. Der kommunikative Austausch, in welcher Form auch immer, darf nicht verloren gehen.

\section{Kontakte zum Austausch? Ein interessantes Stichwort, jetzt, wo die Devise Kontaktreduzie- rung lautet.}

Da haben Sie recht. Das war zu Beginn des ersten Lockdowns auch eine große Herausforderung. In den ersten Wochen war es enorm ruhig und still und es fehlten die Kontake und der kommunikative Austausch. Wir sind nur ein sehr kleines Team, trotzdem haben mir die Lehrer*innen immer wieder mitgeteilt: „Uns gehen die täglichen Treffen sehr ab, mit dir zu reden oder mit den Lehrer*innen untereinander." Wir mussten in den folgenden Monaten schnell lernen, auf vielfältige Weise Kontakt aufzunehmen. Wer hätte gedacht, dass wir binnen kürzester Zeit Online-Konferenzen, Online-Unterricht, Online-Elternabende, Online- Besprechungen durchführen werden? Doch eines ist klar, diese Art und Weise wird niemals den persönlichen Kontakt und Gespräche ersetzen können. 


\section{Auch in den schwierigen Zeiten ist es gelungen, diesen Kontakt aufrechtzuerhalten?}

Auf alle Fälle. In Zeiten der Lockdowns war es besonders wichtig, weiterhin die Verbindung zu allen Kindern und Eltern aufrechtzuerhalten. Mein Team war in dieser Hinsicht sehr engagiert und auch von mir bekamen die Kindern und Eltern mehrmals Post aus der Schule, in der wir zu Gemeinschaftsaktionen aufriefen.

Nachdenklich stimmt in diesen sehr, sehr schwierigen Zeiten der Pandemie dann noch ein Aspekt.

Es braucht Erwachsene, die resilient genug sind, um Kindern stabilen Halt und Zuversicht zu geben. Vor manchen Situationen müssen wir unsere Kinder bewahren und unbedingt als gute Vorbilder vorangehen.

Optimistisch stimmt bei der Auseinandersetzung mit dem Thema der psychischen Widerstandsfähigkeit der Aspekt, dass sich auch die Eltern damit beschäftigt haben und aus unterschiedlichen Perspektiven auf die Förderung der Resilienz der Kinder geachtet wird, dass wir diese Zeit gemeinsam durchstehen, dass es uns allen um die Resilienz der Kinder geht.

\section{Resilienz und Hoffnung, passt das für Sie gut zusammen?}

Diese beiden Wörter passen wunderbar zusammen - die Hoffnung ist mit positiven Erwartungen verbunden, bietet Zuversicht und ist immer auf die Zukunft gerichtet. Gerade in Krisenzeiten spielt Hoffnung eine sehr bedeutende Rolle.

\section{Autorin}

Sabine Höflich, Mag. Dr.

Hochschullehrende an der Pädagogischen Hochschule Niederösterreich, zuvor Volks- und Sonderschul- sowie Ausbildungslehrerin, Arbeitsschwerpunkte: Diversität und Inklusion Förderschwerpunkt soziale und emotionale Entwicklung; Forschung in den Bereichen Resilienz, Autismus und Pädagogisch-praktische Studien.

Kontakt: sabine.hoeflich@ph-noe.ac.at

\section{Quellenhinweis}

Fachstelle NÖ (2021). Gemeinsam stark werden - das Lebenskompetenzprogramm für die Volksschule. https://www.fachstelle.at/portfolio/gemeinsam-stark-werden-programm/ 